\title{
"All my relations": experiences and perceptions of Indigenous patients connecting with Indigenous Elders in an inner city primary care partnership for mental health and well-being
}

\author{
George Hadjipavlou MA MD, Colleen Varcoe RN PhD, David Tu MD, Jennifer Dehoney BHK BSc(PT), \\ Roberta Price, Annette J. Browne RN PhD
}

Cite as: CMAJ 2018 May 22;190:E608-15. doi: 10.1503/cmaj.171390

\begin{abstract}
BACKGROUND: Mental health services in urban settings generally have not been adapted to serve the needs of Indigenous patients. We explored how patients' encounters with Indigenous Elders affected their overall mental health and well-being to identify therapeutic mechanisms underlying improvement.
\end{abstract}

METHODS: We conducted qualitative interviews of participants enrolled in a 6-month prospective mixed-methods evaluation of a program for mental health and well-being that featured the inclusion of Elders in the direct care of Indigenous patients in an inner city pri- mary care clinic. Individual semistructured interviews were conducted to explore patients' experiences and perceptions of their participation in the Elders program.

RESULTS: We included 37 participants from at least 20 different First Nations. All but 1 participant described substantial benefits from their encounters with Elders, and none reported being negatively affected. Five overarching themes were identified: experiencing healing after prolonged periods of seeking and desperation; strengthening cultural identity and belonging; developing trust and opening up; coping with losses; and engaging in ceremony and spiritual dimensions of care as a resource for hope.

INTERPRETATION: Our evaluation illustrates that the Elders program was perceived by participants to have a broad range of positive impacts on their care and well-being. Although this study was based on experiences at a single urban clinic, these findings support the Truth and Reconciliation Commission of Canada's calls to action regarding the inclusion of Elders as a strategy to improve care of Indigenous patients in Canadian health care systems.
M

ental health disparities between Indigenous and nonIndigenous people in Canada are related to underlying economic, social and political inequities that are legacies of colonization and the oppression of Indigenous cultures now recognized as "cultural genocide" by the Truth and Reconciliation Commission of Canada. ${ }^{1-6}$ Therefore, disproportionate rates of mental illness and suicidality must be understood in context, not as intrinsic predispositions of Indigenous people but instead as reflecting persistent inequities, ${ }^{2,7-10}$ This is evident for many Indigenous people living in inner cities where the effects of poverty, racism, and other layers of discrimination and marginalization on mental health are impossible to overlook. . $8,911,12^{2}$

In general, mental health services in urban settings have not been adapted to serve the needs of Indigenous patients, and this is reflected in the comparatively low rates of voluntary utilization, ${ }^{13,14}$ and much higher rates of acute admissions to hospital for suicidality and other mental health crises. ${ }^{15}$ Qualitative studies point to numerous reasons why Indigenous people express reluctance to engage with mainstream health care services: including racism, "being treated as a second-class citizen," and lack of Indigenous staff and cultural practices. ${ }^{8,16-20}$ Ethnographic data suggest that Indigenous people living in the inner city may seek out recognized Elders as informal sources of mental health care in lieu of mainstream services. ${ }^{12}$

There is widespread agreement that First Nations, Inuit and Métis Elders can play a crucial role in the mental health of Indigenous Peoples. ${ }^{1,5,13}$ Elders are recognized by their communities for possessing common qualities that are highly valued - leadership, accumulated wisdom, compassion, community devotion and dedication to personal healing. $5,21,22$ The designation of Elder is achieved by "those who have shown wisdom and leadership in cultural, historical, and spiritual matters within their communities, 
and might not necessarily be old. Elders represent an essential connection with the past; they are keepers of the community knowledge and supporters of its collective spirit."13 Encounters with Elders can provide opportunities for patients to assert or reclaim cultural identity as part of their mental health treatment and reverse the cultural marginalization many Indigenous people have experienced in urban health care settings, such as emergency departments and hospital outpatient programs. ${ }^{13,14}$ One of the Truth and Reconciliation Commission of Canada's "calls to action" is the inclusion of Elders in the treatment of Indigenous patients in Canadian health care systems. ${ }^{1}$

However, Elders have not been formally included or recognized as legitimate care providers within Canadian health care systems, and there have been no previous studies exploring the implementation and impact of an intervention that formally includes Elders in the provision of mental health care for Indigenous patients. Previous research found that involving Elders in a community mental health promotion strategy for teens resulted in a reduction in suicides. ${ }^{23}$ Involving Elders in patient care has also been shown to substantially decrease rates of domestic violence,${ }^{24}$ and to improve understanding and trust between Indigenous and non-Indigenous staff and patients. ${ }^{25}$

Our study is part of a larger investigation examining the impact of an Indigenous Elders program on the mental health and well-being of Indigenous patients in an inner city primary care setting. Quantitative results, which have been reported elsewhere, ${ }^{26}$ showed clinically and statistically significant reductions in depressive symptoms and suicidality. The goal of this study is to explore how encounters with Elders affected patients' overall mental health and well-being to identify the therapeutic mechanisms underlying improvement.

\section{Methods}

\section{Setting}

The study setting was an inner city primary care clinic that has more than 4000 active patients, $65 \%$ of whom identify as Indigenous, from diverse First Nations communities across North America. Many patients have mental health and substance use disorders, chronic pain and other stigmatizing chronic health conditions, such as HIV and hepatitis $C$ virus infections. The primary care team comprised family physicians, nurses, mental health and substance use counsellors, a psychiatrist and medical office support staff. In 2013, the clinic began a partnership program with several Indigenous Elders to provide cultural mentorship to clinic staff and trainees; their roles expanded in 2014 to include direct patient care through one-on-one visits, group cultural teaching circles and seasonal land-based ceremonies. Five Elders worked in direct partnership with the primary care team, participated in clinical rounds and used the clinic's shared electronic medical records. Between 2014 and 2016, more than 300 clinic patients met with 1 or more Elders in the program.

\section{Participants}

This study is part of a larger mixed-methods investigation that followed 41 patients prospectively over 6 months to evaluate the impact of the Elders program. ${ }^{26}$ Study design was developed in consultation with Elders and community advisors. Posters advertising the study were placed in the clinic waiting room and medical offices. Recruitment involved convenience and snowball sampling as we sought to enroll all participants we could identify who met our study criteria. Any patient who self-identified as Indigenous, was older than 18 years and was able to provide informed consent was eligible to participate in the study. Quantitative measures were completed at baseline, 1, 3 and 6 months (further details are published elsewhere ${ }^{26}$ ). Participants who had met with an Elder at least twice at the time interviews were conducted - corresponding to their initial 3 months in the program - were invited to participate in a semistructured interview $(n=28)$. An additional 9 patients who had also met at least twice with the Elders but entered the program before filling out baseline questionnaires (and were therefore ineligible for the longitudinal cohort), were also invited to participate in the interviews, bringing the total sample to 37 patients. There were no specific exclusion criteria. All participants provided written informed consent. Qualitative interviews were conducted over a 4-month period (December 2015 to March 2016), just before the emergence of the "opioid crisis" and associated marked escalation in overdose deaths. ${ }^{27}$

\section{Data collection}

Semistructured, individual interviews were conducted to explore patients' experiences and perceptions of the Elders program. The research protocol and all aspects of data collection were presented at quarterly community gatherings and revised accordingly. The interview protocol was pilot tested in a focus group of Indigenous patients who had experience with the Elders program before the start of the study. Interview questions were modified based on their feedback and review by the Elders (Appendix 1, available at www.cmaj.ca/lookup/suppl/doi:10.1503/cmaj.171390 /-/DC1). Members of the research team who were trained to conduct qualitative interviews interviewed the participants.

\section{Analysis}

All interviews were audiorecorded and transcribed. Transcripts were coded using NVivo 9+ to manage the data. Transcripts were reviewed and discussed in multiple meetings with Indigenous and non-Indigenous members of the research team who conducted a thematic analysis informed by theories of structural violence, decolonization, and trauma-and-violence-informed care. ${ }^{28-32}$

Each investigator was assigned several transcripts, with each transcript assigned to at least 2 (Indigenous and non-Indigenous) investigators. Additionally, the lead investigator read all transcripts. Transcripts were read individually initially, with each investigator identifying preliminary codes. Preliminary analyses and codes were collectively discussed in multiple face-to-face meetings and refined to create a coding guide. Using this guide, a graduate-trained research assistant coded the transcripts under the guidance of the lead investigator. The investigators then analyzed the data within codes, developing preliminary themes both within and across codes, which were further refined through discussions among the larger team. Preliminary analyses and themes derived from the data were presented and discussed at 
community gatherings attended by patients, community members, clinic staff and Elders. These gatherings provided opportunity for stakeholders to review the data and provide feedback to confirm that the identified themes reflected and encompassed their experiences and interpretations. Ethnographic data in the form of field notes by members of the research team who attended community meetings as participant-observers further informed interpretation of interview data. Credibility of the analysis was continually assessed at regular meetings with Indigenous research team members whose lived experience resonated with the patterns and themes identified.

\section{Ethics approval}

We followed Canadian Tri-Council guidelines for ethical research involving First Nations, Inuit and Métis participants. ${ }^{33,34}$ The University of British Columbia and the participating clinic granted ethics approval.

\section{Results}

Thirty-seven patients with diverse Indigenous backgrounds from at least 20 First Nations (12 patients did not provide information about their bands or territories of origin) participated in the study. They ranged in age from 25 to 61 years, $65 \%$ were female, $71 \%$ had a history of foster care, $61 \%$ had a parent who had attended residential school and $27 \%$ had themselves attended residential school.

The 5 Elders came from 4 different territories, which overlapped with the territories of $20 \%$ of all participants. Participants had a median of 7 (interquartile range [IQR] 4-16) visits with Elders.

None of the participants described being negatively affected by their work with Elders. All but 1 participant endorsed some form of benefit from seeing the Elders (this participant expressed feeling optimistic about the potential for benefit but thought that it was too early to tell). Although some participants discussed positive changes that emanated from listening to and directly incorporating the Elders' teachings into their lives, most identified the process of being with Elders - how they related to them or felt in their presence, or what the Elders represented - as the primary source of benefit. We identified 5 overarching but interrelated themes that characterized the participants' experiences of the program.

\section{Theme 1: Finding a place of healing after a prolonged period of seeking and desperation}

Consistent with the experiences of many Indigenous people in Canada, most participants entered the Elders program from a background of severe social adversity and disruption. This was coupled with prolonged periods of seeking "healing" or "recovery" - which they had not found in mainstream health services. Many described high levels of desperation - feeling that they were "breaking down" or "on the verge of giving up."

Recurring descriptions of the ways mainstream health care and associated mental health or addiction services had failed to provide the remedies they needed were contrasted with their experiences in the Elders program. Patients criticized the excessive use of "pills" to treat complex problems, which they often related to poverty and disrupted relationships, and "judgmental" attitudes by health care providers who undervalued patients' knowledge claims, encroached on their agency or were too prescriptive in their treatments. In contrast, patients viewed the Elders as providing a safe "place for healing" where they could "laugh" and receive "gentle guidance" that was nonauthoritarian and "respected" who they were. They repeatedly contrasted previous health care experiences with their genuine, human relationships with the Elders who, by virtue of sharing their own struggles and histories, "accepted" them and "never judged" (Box 1).

Box 1: Patient quotes for theme 1: Finding a place of healing after a prolonged period of seeking and desperation

1. I've gone through counselling before and as I was telling you ... people look down on the Native people as dirty or useless or not up to par with the other communities.... I've always found that that kind of counselling was very detrimental to my mental, emotional well-being. Just being able to sit down with somebody that recognizes you as a human being and also has the cultural education of ... sage and cedar and the natural medicines that biologically [are] part of our universe has made a huge difference for me. [Participant 1]

2. Well to me it's different in a way that she comes from the same background as I do ... the residential school, the breaking of families, of tradition. The loss of language. All ... the struggle to, you know, exist in society. Yeah. So it was almost as if I was coming in out of the cold to realize that this is someplace that feels right to me, that I feel that these people understand me and they have a connection to ... the trails I went down when I thought I was so alone, so ostracized and even abandoned. [Participant 2]

3. You know I can feel that, I get that energy. I get that energy when I go in the room, just the way they sit back and they look at you.... I'm sure that's from probably their own personal experiences because I know [the Elder] ... he's been [through] much the same that I just shared. Which is ... healing for me because I realize, "OK someone else has gone through the same thing as me." And you know l've come to realize that over the years, a lot of people have gone through what I've gone through. [Participant 3]

4. Yeah - cause I've tried like the AA and treatment and everything. It's not really working, so I thought just to get back in touch with Aboriginal culture somehow would help ... [Participant 4]

5. I'm finding it a lot more comfortable coming here anyway because now I have a place just to come to so I can chat with somebody. Because if I am really hurt, I could cry in front of them, and I don't just cry with just anybody, and it makes me feel okay; that I'm not going to be judged or pointed at or laughed at or they're going to go outside and talk about it because they're carrying it and then they have to let it go. So maybe an Elder needs to talk to me sometime. And have you noticed, I use laughter a lot? That's what I've noticed with Elders up north too, they use laughter how to heal. ... The Elders, they know, from listening from their grandparents; they pass it on.

Actually it's calmed me down. That's exactly ... what it is; it's a calming effect that they have on me. I'm less stressed as well after I've seen an Elder. Basically it's medicine that I needed and I know I needed, so when I do really have issues or I'm feeling blue, it makes me happy to see them. [Participant 5] 
Theme 2: Strengthening cultural identity and belonging Many patients linked the positive health impacts of the Elders program to strengthening their sense of cultural identity and belonging as Indigenous people. Improvements in self-esteem and selfconfidence were described as corollaries of developing a stronger sense of cultural identity, which for some participants encompassed feelings of pride that they had not experienced previously. Participants often alluded to how their own histories of cultural oppression had an enduring, disruptive effect on their sense of self and overall well-being, previously hindering efforts to improve their health, as these facets of their experience had remained unaddressed. They highlighted the importance of talking with Elders with whom they shared histories of colonial oppression, and how doing so enabled them to "address the more deeprooted problems" underlying their symptoms or distress.

For many participants, the experience of feeling "connected" to an Elder became a bridge to other relationships or communi-

Box 2: Patient quotes for theme 2: Strengthening cultural identity and belonging

1. And meeting with [the Elders] has given me this: "It's OK to be Native. It's OK to be Native." Like I know that, but they reinforced that. They did, they reinforced it. "[Patient name], it's OK to be Native." And again I got so much respect for both of them - the way they sit and the way they talk and the way they come across to you. You can't help but respect them. They're two very proud people and that's something that I have to work at is being more proud of myself, and respect myself more. Which I believe I'm doing by reaching out. I've reached out. I've come out of denial. I'm asking for help. I've done it a number of times, but I don't think it was wholehearted like this.

I got - uh, what do you call it? - an identity crisis. I've got abandonment issues. I've got a lot of guilt and shame that I'm hanging on to and, like I said, those resentments. And I know that I can come here and deal with some of those things.... This is a chance to sit down and be more intimate and personal with somebody. Somebody who I know understands. And is of the same race, which was important to me because I wanted to connect more and be closer to my community. [Participant 3]

2. ... It's given me a little more belief and faith in myself. Even though I have had a couple minor relapses since, they've been minor. I haven't gone out and done the full-blown. I'm addressing some things with [the Elder] as well as [another Elder] in the session. I was able to talk about some things that were eating at me. Has it made a difference? Yes it has.... I've got a good place to come to, you know, to discuss these problems that I have. I know these people care, you know. And that feels good. It's given me more confidence. It gave me a little bit more self-esteem. It's healed me a little bit. [Participant 3]

3. I find that learning to love myself and respecting myself and setting healthy boundaries for myself, I've never done that, and I've never asked anyone to help me through this. [Participant 6]

4. Again being able to stand in front of somebody that does have [that background]. Well they've gone through everything from foster care, residential school, alcoholism in the family. I mean when they started stealing the children, nobody grew up with how to be a parent or an adult, so having that background makes connecting with the Elders so much easier. We can talk about those things and there's a common ground. [Participant 1] ties, providing a "sense of belonging" or being "part of something" that mitigated painful experiences of isolation or loneliness. As 1 participant put it, spending time with the Elder was about trying "to heal that thing that's keeping me away from being with my people." Participants also frequently described the Elders as surrogates for family - grandparents, parents, aunts - those they had lost because of death or disconnection from their communities of origin (Box 2).

\section{Theme 3: Developing trust and "opening up"}

Despite attending the clinic for many years, and often speaking favourably about clinicians and staff, many patients described "opening up" about their spiritual or mental health struggles only after the start of the Elders program. Participants frequently described "trusting" the Elders, which they contrasted with mistrust of the health care system and wariness about learning from health care providers. A related theme was that, for many participants, therapeutic experiences of trust with the Elders facilitated a parallel process of "opening up" - becoming receptive - to other aspects of their medical or mental health care. Increased openness derived from trust in their relationship with the Elders extended to openness to learning more generally - to learning from other relationships or knowledge. Patients talked about no longer perceiving aspects of their social worlds from a perspective of mistrust, enabling them to "reach out" and engage more meaningfully with supports or treatment.

Conversely, a few participants had established trusting long-term relationships with their physicians and sought out the Elders because of the physicians' recommendations or encouragement. Some of these participants described a history of disconnection from their Indigenous communities because of traumatic past experiences and, that for them, connecting with Elders represented "opening up" not to mainstream medicine but to their own Indigenous cultures (Box 3).

\section{Theme 4: Coping with losses}

Most participants experienced multiple losses and deaths in their families and social worlds, including loss of community, language, identity and connection to their culture. Patients frequently acknowledged how, as Indigenous people living in the inner city, they experienced a disproportionate number of losses, often at a young age, without access to meaningful supports. As 1 participant summarized, "everybody down here has the same story ... of hurt, pain, loss." Participants who struggled with substance use readily linked relapses to losses and viewed resorting to drug use as an available strategy to alleviate grief or emotional pain for which they had been unable to find solutions in the health care system.

Participants described the Elders as offering ways - teachings, support, ceremonies - to allow them to bear these losses. They viewed the Elders as having the "wisdom and knowledge about life" to provide the guidance they needed to cope with grief. Participants also perceived the Elders as models of resilience who had overcome - and continue to overcome - histories of profound adversity, especially in the form of cumulative losses (Box 4). 
Theme 5: Engaging in ceremony and spiritual dimensions of care as a resource for hope

Seeking spiritual guidance was a common reason for seeing an Elder. Although participants frequently referred to the inseparability of their "spirit" and their emotional or mental health and that spiritual struggles underpinned more overt symptoms of distress, they also drew sharp distinctions about where, how and by whom their spiritual care could be addressed. They readily identified spiritual dimensions to their physical or mental health concerns but did not believe their health care providers could address these aspects of care ("I see the doctor about health things - not spiritual things").

Conversely, meetings with the Elders typically involved some form of ceremony or prayer. Although this could be overt, in the form of smudging, drumming or brushing, for many patients sim-

Box 3: Patient quotes for theme 3: Developing trust and "opening up"

1. I can talk and trust and not feel worried about her saying anything to anybody and because, like growing up, like our trust - like my trust - has been broken in many areas. And so you know just coming to see somebody is really hard for me to open up ... [The Elder] had like a ... kind, soft-spoken [voice] and just welcomed me in. ... It is very hard for an Aboriginal woman or a person to be open for a lot of things ... I trust [The Elder] to talk to her about these things. And like I said: it's like people are put in our path for a reason. And she's the teacher and I am learning from her. Or else she is learning from me and I am teaching her something. [Participant 7]

2. I wasn't able to ask for assistance or guidance ... my guards were up toward people, because I was seeing things and I couldn't ... comprehend the feeling that I had toward some people. And my trust issues were really high, not trusting some people because I was [thinking], "I know they are not going to help me." [The Elder] really helped me through talking, just sitting and talking and just brought my spirit back. I'm able to dress up now again, because I couldn't dress up [before]. [Participant 6]

3. I mean you can't get help unless you ask for it, right. So that's what I'm starting to do is open up to people instead of shutting myself down because l'm finding that opening myself up things are actually starting to happen other than before I'd say something, say there's a problem but I wouldn't get deep into it and these people [doctors] don't know what's really going on, they know there's a problem but ... they don't know what the problem is so how can they help you? [Participant 8]

4. You know, I used to come in here drunk, ranting and raving? Kicked out a few times. Wasn't happy with myself. Didn't know where I fit in yet in Vancouver. [The receptionist] used to just hide under the table when he saw me coming ...। respect the premises a lot more now, I realize that each and every individual, even the people that come here to the circle are here to help. They help you because they're just like me, you know, they're healing. When you're talking, teaching, you're healing. Everybody downstairs, they're there for you. You got the mental health part as well as a team of fantastic doctors ... and the office staff are there to help you. I find [the receptionist] now what a great guy - what was I doing hollering at him? It's not his fault they've been backlogged for 2 hours. ... So I learned to sit and be quiet and be patient. [Participant 3] ply "sitting with" the Elder enabled them to feel that they were "taking care of [their] spirit." For those participants who had grown up in non-Indigenous families, participating in ceremonies represented new cultural experiences; for others, engaging with Elders reawakened ceremonies and traditions from their pasts that had remained dormant (1 patient reported that $25-30$ years had elapsed since she had participated in ceremony). Because participants came from diverse backgrounds and disparate communities, most did not share the same language or traditions of the Elders at the clinic. However, participants readily identified the Elders' spiritual teachings, prayers and ceremonies as the "common ground" of a broader shared Indigenous history and a direct way of "connecting with culture." Participants recounted how attending to spiritual matters and making ceremonies part of their lives restored hope (Box 5).

\section{Interpretation}

A growing body of research shows that Indigenous people continue to experience discrimination and dismissal when attempting to access health care services, which is often compounded for those living with serious mental health or substance use issues. ${ }^{8,17,35-38}$ Many urban Indigenous patients avoid mainstream health services, which they perceive to be culturally unsafe, and when they do access services, they have limited trust and engagement with health care providers. ${ }^{37-39}$ To address these pressing problems, in a partnership between Indigenous Elders and western-trained health care providers, we undertook

\section{Box 4: Patient quotes from theme 4: Coping with losses}

1. It cleared up a lot of things about growing up and life and losing my grandparents and losing my mom and just being mentally stable and not going nuts and committing suicide. Breaking up with my girlfriend and having 2 daughters, but different mothers. And talking to [the Elder] about all this and she would say, "I understand." I didn't have my mom anymore, and she was the [person] I talked to about these things. But yeah, I loved it, just talking with her. [Participant 9]

2. I lost my dad, and now my grandma and my cousins. It's like crazy. I just felt like after she talked with me it felt like I can release my dad and let him go. Like he's saying, "I'm okay, let me sleep now," and that's how I felt that day. Now I'm doing the same thing on my grandma and all of them. My cousin turned around and came down to me the other day and said, "I'm fine, leave me alone, I'm fine; he's taking care of me." They came in my dreams. I'm like, "Oh my God, they're starting to come down to see me." [Participant 10]

3. My experience with the Elders program has had a huge impact. Like I said, I went through grief and loss in the last 2 years, and losing my twin brother was the hardest so ... I asked for more help to cleanse my home and to have drummers and ... to keep going on the right path. [Participant 7]

4. That changed a lot because I'm not thinking of suicide anymore. ... and that's another reason why I came here because I'd been so suicidal lately since the death of my kids and anniversary of their death. I didn't know who to go to, who to talk to. [Participant 11] 
a "cultural intervention" of including Indigenous Elders in the provision of care. Our qualitative evaluation suggests that this intervention was effective, with a broad range of positive impacts. These qualitative findings complement our quantitative investigation showing substantial reductions in depression and suicidal ideation. ${ }^{26}$

Box 5: Patient quotes for theme 5: Engaging in ceremony and spiritual dimensions of care as a resource for hope

1. I just see the doctor about health things - not about spiritual things and mental things and psychological things. Even though he always asks me, "How do you feel?" about stuff. "What do you feel about that?" I said, "I just don't want to talk. I'm fine." [Participant 9]

2. ... Ever since [Elder name] did the brushoff [traditional practice] for me, I was able to go out. I finally went out. All the years I've been dreaming about going out to get cedar [land-based tradition] and I finally went out and got cedar; went out and I got so much cedar. ... He made me really see that I'm okay, there is nothing wrong with me unless I do something bad then somebody would say something, if I did something bad. Because all my life I grew up - that little girl - I thought I was a bad person. [Participant 6]

3. ... I love the prayers that we would start off and end with. That brought comfort and soothing, healing my soul. That's what she brought to me. Yeah so l've been doing a lot more [traditional ceremonial] cleansing of the house ... since I started seeing [the Elder]. Cause before it was only when I saw [the Elder] I got my cleansing done, but now I think I do it every 2 days. I wake up and I cleanse myself. [Participant 12]

4. Yes, hopefulness. And that's a very important word ... when I walked out I felt hopefulness rather than having pills thrown at me - you know, that our emotional struggles that we encounter in life such as racism, you know, our terrible parents or sexual abuse or whatever it may be, it's being able to let a little bit out makes the day go better... I went through stage 4 cancer and my oncologist basically said I had a $20 \%$ chance of living for 5 years. I'm in my fifth year right now, and so for the last 5 years I've been waiting to die and not doing rituals and not having hope to live, just kind of waiting like, "OK so when's it going to kill me?" ... I have no cancer now but getting over that hump, that mental block, this Elder program has changed - like changed my life. I'm willing to live now. I'm not looking at when am I going to die it's like, "Well how much life can I live?" So it has a huge impact.

... I've never experienced something as positive when it comes to my spiritual, emotional well-being. That having this kind of [Elder] support is life changing.... [Participant 1]

5. ... I felt suicidal before I started coming here. It was after I started talking ... everything just started coming together. I felt suicidal because I didn't know what the hell I was going to do, right. ... It helped me to talk about everything. And I haven't thought about dying. [Laughs] Six months now I haven't thought about suicide or anything. [Participant 13]

6. I feel more relaxed in my life today. Whenever I feel depressed I always say to myself, "Focus, concentrate, go for a walk, do something positive," and it works. I pray, I smudge, I try to do the right things every day, to be kind, to love other people because it's not their fault. Just being patient. Take care of myself. Love myself more. That's about the only things I can think of right now. [Participant 14]
Participants reported that engaging with Elders allowed them to address unmet needs in their health care and overall wellbeing, especially in the form of attending to underlying spiritual issues, strengthening cultural identity and addressing the enduring negative impacts of cultural oppression and historical trauma. The concept of historical trauma has been used to refer to the collective, cumulative and complex intergenerational impacts of colonization (e.g., residential school, foster care and other profoundly disruptive effects of government policies). ${ }^{40}$ Gone proposed that the treatment of choice for historical trauma is not a selective serotonin reuptake inhibitor or evidence-based psychotherapy for posttraumatic stress disorder, but participation in cultural practices - a view that is also espoused by the Elders in the program. ${ }^{40}$ Access to Elders as part of routine primary care offers one important avenue for meaningful participation in cultural practices that can improve Indigenous patients' care and help reduce inequities. Our findings are consistent with research showing that the inclusion of Elders in health care initiatives led to a reduction in teen suicides, ${ }^{23}$ decreased rates of domestic violence, ${ }^{24}$ improved quality of life, reduced depressive and trauma symptoms, ${ }^{41}$ and improved understanding and trust between Indigenous and non-Indigenous staff and patients. ${ }^{25}$

Our research suggests that working with Elders allowed some participants to "open up" and become more receptive to engaging with other health care providers. Most participants entered the Elders program from a position of epistemic mistrust in their interactions with the health system - a pervasive wariness about trusting others as a source of knowledge that would be relevant or helpful to them. ${ }^{42}$ For many Indigenous patients, the legacies of colonization have left a deep epistemological chasm that cannot be easily traversed by health care professionals, no matter how well intended or culturally competent. ${ }^{43}$ Participants repeatedly emphasized the importance of shared histories of colonial oppression as a "common ground" for building trust and openness with the Elders, enabling them to establish therapeutic alliances they had not previously experienced with health care providers. In this way, relational experiences of trust with the Elders seemed to lessen epistemic mistrust that previously acted as an obstacle to care.

Many patients repeatedly emphasized therapeutic experiences of feeling listened to and accepted for "who they were," which they contrasted with previous experiences of having their concerns medicated away ("pills thrown at me"). They frequently described their positive therapeutic experiences with the Elders in terms that map onto the common factors underpinning evidence-based therapeutic relationships (e.g., therapeutic alliance, genuine empathy, positive regard), ${ }^{44,45}$ suggesting 1 possible mechanism that reconciles the Elders' "cultural practice" with evidence-based therapies. ${ }^{40,46}$

The instillation of hope and alleviation of suicidal ideation experienced by many of the participants in this study through their engagement with Elders was often linked to strengthening cultural identity and reestablishing cultural connections. This is consistent with previous research showing that cultural continuity, which encompasses active efforts to maintain or revitalize cultural institutions and practices within Indigenous communities, is a strong community-level protective factor against suicide. ${ }^{47-49}$ 
Our findings also suggest that the presence of Elders as legitimate alternatives to seeing physicians or other mainstream clinicians had a positive disruptive effect on the "medical culture" of the clinic, narrowing perceived power inequalities. This is in line with previous research showing that the confidence of Indigenous Peoples in the health care system could be renewed by positive therapeutic relationships that shifted power imbalances. ${ }^{38}$

\section{Limitations}

This study was conducted in an urban clinic that has an explicit mandate to serve Indigenous and highly marginalized people. Hence, there was an existing level of commitment and openness to integrating Indigenous culture. Although the findings may be applicable in more conventional settings, the influence of specific health care organizations, policies and wider social contexts would need to be taken into account.

\section{Conclusion}

Our findings support the Truth and Reconciliation Commission of Canada's calls to action regarding the inclusion of Elders in the treatment of Indigenous patients in Canadian health care systems. By attending to underlying spiritual issues, strengthening cultural identity and addressing the enduring negative impacts of cultural oppression and historical trauma, encounters with Elders provided a therapeutic mechanism to improve health outcomes. Meaningful awareness of the historical and ongoing colonial context in which we all live and Indigenous patients seek to thrive is integral to the need, success and challenges faced by the Elders program - and other health care initiatives aimed at improving the health of Indigenous people. Although such programs are essential to support health and healing, without attending to the upstream roots of health disparities experienced by Indigenous people that are evident in our findings, such disparities will persist. Thus, we suggest that there is a need for Indigenous leadership when planning programming for Indigenous people in partnership with mainstream health care services.

\section{References}

1. Honouring the truth, reconciling for the future: summary of the final report of the Truth and Reconciliation Commission of Canada. Winnipeg: Truth and Reconciliation Commission of Canada; 2015.

2. Adelson N. The embodiment of inequity: health disparities in Aboriginal Canada. Can J Public Health 2005;96:S45-61.

3. Brown HJ, McPherson G, Peterson R, et al. Our land, our language: connecting dispossession and health equity in an indigenous context. Can J Nurs Res 2012; 44:44-63.

4. Kirmayer LJ, Simpson C, Cargo M. Healing traditions: culture, community and mental health promotion with Canadian Aboriginal peoples. Australas Psychiatry 2013;11:15-23.

5. Report of the Royal Commission on Aboriginal Peoples. Ottawa: Royal Commission on Aboriginal Peoples; 1996.

6. Gracey M, King M. Indigenous health part 1: determinants and disease patterns. Lancet 2009;374:65-75.

7. Waldram JB. Revenge of the Windigo: the construction of the mind and mental health of North American Aboriginal Peoples. Toronto: University of Toronto Press; 2004.

8. Goodman A, Fleming K, Markwick N, et al. "They treated me like crap and I know it was because I was Native": The healthcare experiences of Aboriginal peoples living in Vancouver's inner city. Soc Sci Med 2017;178:87-94.
9. Browne AJ, Stout MD. Moving towards Nahi: addressing health equity in research involving indigenous people. Can J Nurs Res 2012;44:7-10.

10. Jongbloed K, Pearce ME, Pooyak S, et al. The Cedar Project: mortality among young Indigenous people who use drugs in British Columbia. CMAJ 2017;189: E1352-9.

11. Benoit C, Carroll D, Chaudhry M. In search of a healing place: Aboriginal women in Vancouver's Downtown Eastside. Soc Sci Med 2003;56:821-33.

12. Culhane D. Narratives of hope and despair in Downtown Eastside Vancouver. In: Kirmayer LJ, Valaskakis G, editors. Healing traditions: the mental health of Aboriginal Peoples in Canada. Vancouver: UBC Press; 2009:160-77.

13. King M, Smith A, Gracey M. Indigenous health part 2: the underlying causes of the health gap. Lancet 2009;374:76-85.

14. Kirmayer LJ, Tait C, Simpson C. The mental health of Aboriginal peoples in Canada: transformations of identity and community. In: Kirmayer LJ, Valaskakis G, editors. Healing traditions: the mental health of Aboriginal peoples in Canada. Vancouver: UBC Press; 2009:3-35.

15. Pathways to health and healing - 2nd report on the health and wellbeing of Aboriginal peoples in British Columbia. Victoria: Office of the Provincial Health Officer; 2009.

16. Menzies P, Bodnar A, Harper V. The role of the Elder within a mainstream addiction and mental health hospital: developing an integrated paradigm. Native Soc Work J 2010;7:87-107.

17. Browne AJ, Varcoe CM, Wong ST, et al. Closing the health equity gap: evidencebased strategies for primary health care organizations. Int J Equity Health 2012;11:59.

18. Browne AJ, Smye VL, Rodney P, et al. Access to primary care from the perspective of Aboriginal patients at an urban emergency department. Qual Health Res 2011;21:333-48.

19. Tang SY, Browne AJ. 'Race' matters: racialization and egalitarian discourses involving Aboriginal people in the Canadian health care context. Ethn Health 2008;13:109-27.

20. Varcoe C, Browne AJ, Wong S, et al. Harms and benefits: collecting ethnicity data in a clinical context. Soc Sci Med 2009;68:1659-66.

21. Ellerby JH. Working with indigenous elders: an introductory handbook for institutionbased and health care professional based on the teachings of Winnipeg-area aboriginal elders and cultural teachings. Winnipeg: Native Studies Press; 2006.

22. Mehl-Madrona L. What traditional indigenous elders say about cross-cultural mental health training. Explore (NY) 2009;5:20-9.

23. Neligh G. Mental health programs for American Indians: their logic, structure and function. Am Indian Alsk Native Ment Health Res Monogr Ser 1990;3:1-279.

24. Puchala C, Paul S, Kennedy C, et al. Using traditional spirituality to reduce domestic violence within aboriginal communities. J Altern Complement Med 2010;16:89-96.

25. Cook SJ. Use of traditional Mi'kmaq medicine among patients at a First Nations community health centre. Can J Rural Med 2005;10:95-9.

26. Tu D, Hadjipavlou G, Browne AJ, et al. Partnering with Indigenous Elders in primary care improves mental health outcomes of inner-city Indigenous patients - a prospective cohort study. Can Fam Physician. In press.

27. Provincial health officer declares public health emergency [news release]. $B C$ Gov News 2016 Apr.14. Available: https://news.gov.bc.ca/10694 (accessed 2017 Sept. 30).

28. Braun V, Clarke V. Using thematic analysis in psychology. Qual Res Psychol 2006;3:77-101.

29. Farmer PE, Nizeye B, Stulac S, et al. Structural violence and clinical medicine. PLoS Med 2006;3:e449.

30. Martin DH. Two-eyed seeing: a framework for understanding indigenous and non-indigenous approaches to indigenous health research. Can J Nurs Res 2012;44:20-42.

31. Braun KL, Browne CV, Ka'opua LS, et al. Research on Indigenous Elders: from positivistic to decolonizing methodologies. Gerontologist 2014;54:117-26.

32. Oral R, Ramirez M, Coohey C, et al. Adverse childhood experiences and trauma informed care: the future of health care. Pediatr Res 2016;79:227-33.

33. Tri-Council Policy Statement (TCPS2): Ethical conduct for research involving humans. In: Research involving the First Nations, Inuit and Métis peoples of Canada. Ottawa: Canadian Institutes of Health Research, Natural Sciences \& Engineering Research Council of Canada, Social Science \& Humanities Research Council of Canada; 2014. 
34. Wright AL, Wahoush O, Ballantyne M, et al. Qualitative health research involving indigenous peoples: culturally appropriate data collection methods. Qual Rep 2016;21:2230-45.

35. Smye V, Browne AJ, Varcoe C, et al. Harm reduction, methadone maintenance treatment and the root causes of health and social inequities: an intersectional lens in the Canadian context. Harm Reduct J 2011;8:17.

36. Browne AJ, Varcoe C, Ford-Gilboe M, et al. EQUIP healthcare: an overview of a multi-component intervention to enhance equity-oriented care in primary care settings. Int J Equity Health 2015;14:152.

37. Browne AJ, Varcoe C, Lavoie J, et al. Enhancing health care equity with Indigenous populations: evidence-based strategies from an ethnographic study. BMC Health Serv Res 2016;16:544.

38. Jacklin KM, Henderson RI, Green ME, et al. Health care experiences of Indigenous people living with type 2 diabetes in Canada. CMAJ 2017;189:E106-12.

39. Allan B, Smylie J. First peoples, second class treatment: the role of racism in the health and well-being of Indigenous people in Canada. Toronto: Wellesley Institute; 2015. Available: www.wellesleyinstitute.com/wp-content/uploads/2015/02/ Summary-First-Peoples-Second-Class-Treatment-Final.pdf (accessed 2017 Nov. 17).

40. Gone JP. Redressing First Nations historical trauma: theorizing mechanisms for indigenous culture as mental health treatment. Transcult Psychiatry 2013;50:683-706.

41. Varcoe C, Browne AJ, Ford-Gilboe M, et al. Reclaiming our spirits: development and pilot testing of a health promotion intervention for Indigenous women who have experienced intimate partner violence. Res Nurse Health 2017;40: 237-54.

42. Fonagy $\mathrm{P}$, Allison $\mathrm{E}$. The role of mentalizing and epistemic trust in the therapeutic relationship. Psychotherapy (Chic) 2014;51:372-80.

43. Wendt DC, Gone JP. Rethinking cultural competence: insights from Indigenous community treatment settings. Transcult Psychiatry 2012;49:206-22.

44. Norcross JC, Wampold BE. Evidence-based therapy relationships: research conclusions and clinical practices. Psychotherapy (Chic) 2011;48:98-102.

45. Norcross JC, Lambert MJ. Psychotherapy relationships that work II. Psychotherapy (Chic) 2011;48:4-8.

46. Gone JP. Reconciling evidence-based practice and cultural competence in mental health services: introduction to a special issue. Transcult Psychiatry 2015;52:139-49.

47. Chandler MJ, Lalonde CE. Cultural continuity as a hedge against suicide. Transcult Psychiatry 1998;35:191-219.

48. Chandler MJ, Lolande CE. Cultural continuity as a moderator of suicide risk among Canada's First Nations. In: Kirmayer LJ, Valaskakis G, editors. Healing traditions: the mental health of Aboriginal peoples in Canada. Vancouver: UBC Press;2009:221-48.

49. Bodnar A. Perspectives on Aboriginal suicide: Movement toward healing. In: Menzies P, Lavallée LF, editors. Journey to healing: Aboriginal people with addiction and mental health issues. Toronto: CAMH Publications; 2014.

\section{Competing interests: None declared.}

This article has been peer reviewed.

Affiliations: Department of Psychiatry (Hadjipavlou), School of Nursing (Varcoe, Browne, Price), Department of Family Practice (Tu), University of British Columbia, Vancouver, BC; Member of the Missanabie Cree First Nation (Dehoney), Missanabie, Ont.; Member of the Snuneymuxw, Nanaimo, BC and Cowichan First Nations (Price), Duncan, BC
Contributors: George Hadjipavlou contributed to the methodological design, data collection, analysis and interpretation, and led manuscript development. Colleen Varcoe, David Tu, Annette Browne and Jennifer Dehoney contributed to the methodological design, data collection, analysis and interpretation, and manuscript development and review. Roberta Price contributed to the methodological design, data analysis and interpretation, and manuscript review. All of the authors approved the final version to be published and agreed to be accountable for all aspects of the work.

Funding: Canadian Institutes of Health Research, Pathways to Health Equity for Aboriginal Peoples Grant.

Accepted: Mar. 2, 2018

Correspondence to: George Hadjipavlou, hadj@mail.ubc.ca 